\title{
Gradhiva
}

GRADHIV

Revue d'anthropologie et d'histoire des arts

12 | 2010

La musique n'a pas d'auteur

\section{Nimbuda ou la carrière d'un citron amer}

Musiques régionales et industrie cinématographique en Inde

Nimbuda or the Bitter Lemon's Trajectory. Regional Music and the Film Industry in India

\section{Christine Guillebaud}

\section{OpenEdition}

Journals

Édition électronique

URL : http://journals.openedition.org/gradhiva/1847

DOI : $10.4000 /$ gradhiva. 1847

ISSN : $1760-849 x$

\section{Éditeur}

Musée du quai Branly Jacques Chirac

Édition imprimée

Date de publication : 24 novembre 2010

Pagination : $56-79$

ISBN : 978-2-35744-029-6

ISSN : 0764-8928

\section{Référence électronique}

Christine Guillebaud, « Nimbuda ou la carrière d'un citron amer », Gradhiva [En ligne], 12 | 2010, mis en ligne le 24 novembre 2013, consulté le 20 avril 2019. URL : http://journals.openedition.org/ gradhiva/1847 ; DOl : 10.4000/gradhiva.1847 


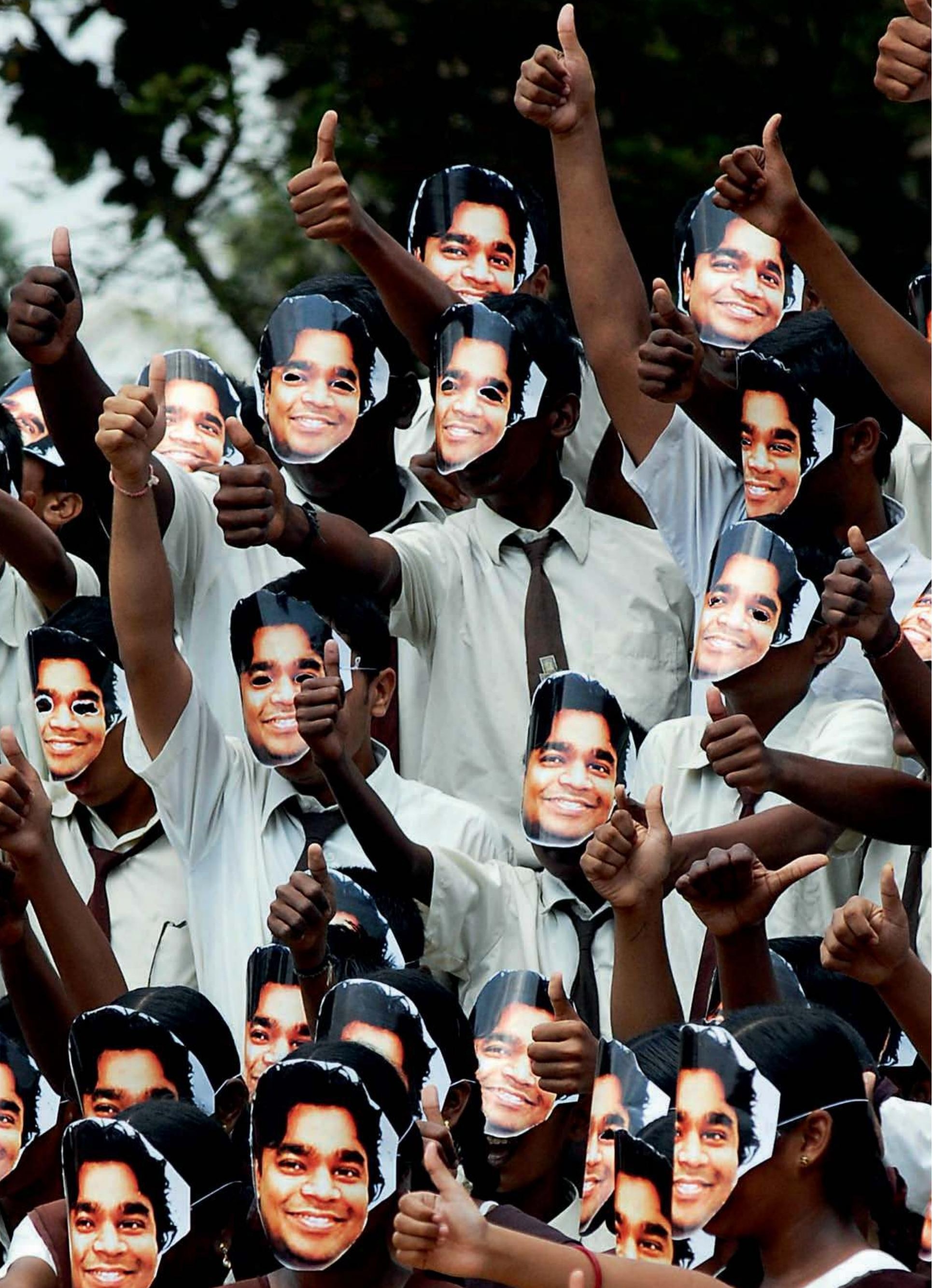


Christine Guillebaud

\footnotetext{
- $\bullet$

1. La notion d'emprunt est utilisée ici dans son acception large, c'està-dire comme une opération de transfert. L'étymologie du terme pose problème. Elle suppose l'idée de propriété qui ne va pas toujours de soi dans les opérations musicales effectuées.

2. Ce projet, coordonné dès 1988 par J. Peter Burkholder et David C. Birchler à l'université de Wisconsin-Madison, s'est ensuite poursuivi à l'université d'Indiana (Giger 1994 : 871).
}

3. À titre d'exemple, Burkholder dresse une typologie de quatorze procédés différents dans l'œuvre de Charles Ives (1994 : 854), dont certains étaient déjà largement utilisés dans les musiques de la Renaissance et du romantisme.

4. Voir par exemple, pour le jazz, les sept « traitements » d'un standard analysés par Patrick Williams (2006).

\section{${ }_{\text {ou }}$ Nimbuda \\ ou Nimbuda la Carrière d'un citron amer Musiques régionales et industrie
cinématographique en Inde Musiques régionales et industrie
cinématographique en Inde r}

Il est une procédure de création musicale particulièrement répandue, l'emprunt de musique existante. Elle consiste pour le musicien ou le compositeur à s'appuyer sur des matériaux préexistants et à les réutiliser pour produire des pièces nouvelles ${ }^{1}$. Selon les époques et les genres musicaux, nombre de ces emprunts ont été documentés, en particulier pour l'Europe de la Renaissance et du xix siècle, pour le be-bop ou encore chez certains compositeurs contemporains comme Charles Ives (Burkholder 1995) ou Luciano Berio (Osmond Smith 1985). L'impressionnante bibliographie annotée et réunie par le Center for the History of Music Theory and Literature de l'universite $d^{\prime}$ Indiana ${ }^{2}$ recense plusieurs centaines de références sur cette procédure, des musiques du xiII siècle aux genres populaires du xxi siècle en passant par le jazz et le classique romantique. Comme le montre aussi cette étude, l'emprunt n'est pas unique en soi, ni même réductible à des prototypes de styles ou d'époques. Il prend au contraire des formes particulièrement diverses, en témoigne le nombre de termes pour le désigner, tantôt citation et référence, tantôt imitation, allusion, paraphrase, etc. ${ }^{3}$ Enfin, il est généralement porteur de propos extramusicaux et d'effets très divers 4 .

J'aborderai ici le cas précis de l'emprunt de mélodie, un procédé des plus ordinaires et des plus généralisés en Inde. Il consiste à utiliser un ou plusieurs airs préexistants, de manière partielle, totale et/ou combinée, et à les investir d'un nouveau contenu, textuel, stylistique, esthétique, etc. C'est généralement la définition qu'en donnent les travaux académiques portant sur les processus de création musicale, mais aussi plus largement les amateurs de musique «indienne». On pense en particulier aux fans de chansons de films (filmi music), l'une des industries culturelles les plus florissantes au monde. Ces emprunts sont connus 
PAGE 56

Fig. 1 Étudiants et fans du compositeur A.R. Rahman à Chennai le 23 février 2009 au moment de la nomination du compositeur aux Oscars. Photo STR @ STR/AFP.

\footnotetext{
-

5. Le fait de laisser les principaux protagonistes dans l'anonymat permettra de considérer Nimbuda comme un exemple emblématique plutôt que comme un cas singulier.
}

sous le nom de «parodies », "versions » (Manuel 1993 : 131-152), ou encore de « recyclage " (Marcus 1993) ou de remake. Dans les pages qui suivent, il ne s'agira pas d'analyser l'emprunt mélodique dans ses spécificités «indiennes», bien qu'il eût été intéressant d'intégrer aussi ce type d'exemple non occidental au corpus de l'université américaine d'Indiana. Je montrerai plutôt comment celui-ci surgit de manière nouvelle dans les débats portant sur le statut juridique des musiques concernées. En effet, envisagé récemment à travers les notions d'« œuvre ", d'«auteur» et de "propriété ", le procédé ouvre un champ d'investigation quelque peu différent : comment questionner la notion juridique de "propriété intellectuelle» face à des pratiques cultivant l'emprunt de mélodies? Comment ont émergé les controverses relatives au droit d'auteur alors que celui-ci semblait jusqu'ici étranger aux manières locales de créer? Comment s'en accommodent aujourd'hui les compositeurs et les musiciens locaux à différents niveaux de leur pratique, à la fois d'un point de vue esthétique et dans le rapport qu'ils entretiennent aux institutions qui les encadrent et les promeuvent?

Pour tenter d'y répondre, je partirai d'une étude de cas, la chanson Nimbuda, "Citron amer" en hindi. Emblématique des controverses dont nous parlons, elle fut attribuée à la fin des années 1990 à différents "auteurs", tour à tour un chanteur de basse caste manganiyar [A] puis un compositeur de musiques de films de Bollywood $[\mathrm{B}]^{5}$. La chanson en a hérité une carrière des plus singulières. Air de musicien professionnel d'un village rajasthani transporté dans les studios de Bollywood en passant par les archives sonores des chercheurs, les scènes de festivals de musiques du monde ou encore les milieux de la jet set de Bombay, le Citron amer a porté et fait sonner des discours contradictoires. Si notre exemple semble a priori éloigné d'une symphonie de Gustav Mahler ou de Berio, il repose bel et bien sur un même principe créatif d'emprunt. Cependant, étant bien plus enclin à circuler dans des mondes contrastés - celui des musiciens-généalogistes, des producteurs de films, des médiateurs culturels et des intellectuels -, son statut (juridique) s'en trouve d'autant plus difficile à déterminer. Avant d'analyser la controverse née autour de la chanson Nimbuda, explorons tout d'abord les termes du débat.

\section{Copie, plagiat, parodie, remake}

Que signifie emprunter une mélodie et que recouvre cette opération dans différents milieux de production musicale en Inde? Pour les ethnomusicologues, un constat commun s'impose : l'emprunt est de règle dans de nombreux genres musicaux, dans les musiques classiques comme dans nombre de traditions régionales. Il révèle combien les chanteurs cultivent un goût du renouvellement et ne cessent d'élargir leurs stocks de possibles pour réaliser de manière différente une même pièce. Ce procédé s'inscrit en outre dans une situation de cohabitation sociale des plus éclatées : les musiciens spécialistes d'une caste empruntent aux répertoires d'autres castes - les généalogistes aux barbiers, les astrologues aux exorcistes -, sans que l'identité de chacun des groupes en présence s'en trouve au final confondue. En effet, je parlerais plus volontiers de transfert que d'emprunt là où le musicien déplace une mélodie sans forcément conserver ce qui fait la signature ou la marque de fabrique du répertoire auquel il puise : les énoncés textuels, les techniques vocales et ornementales, ou plus généralement l'expression. Il transfère ici un air dénué de ses traits stylistiques qui, eux, restent toujours 


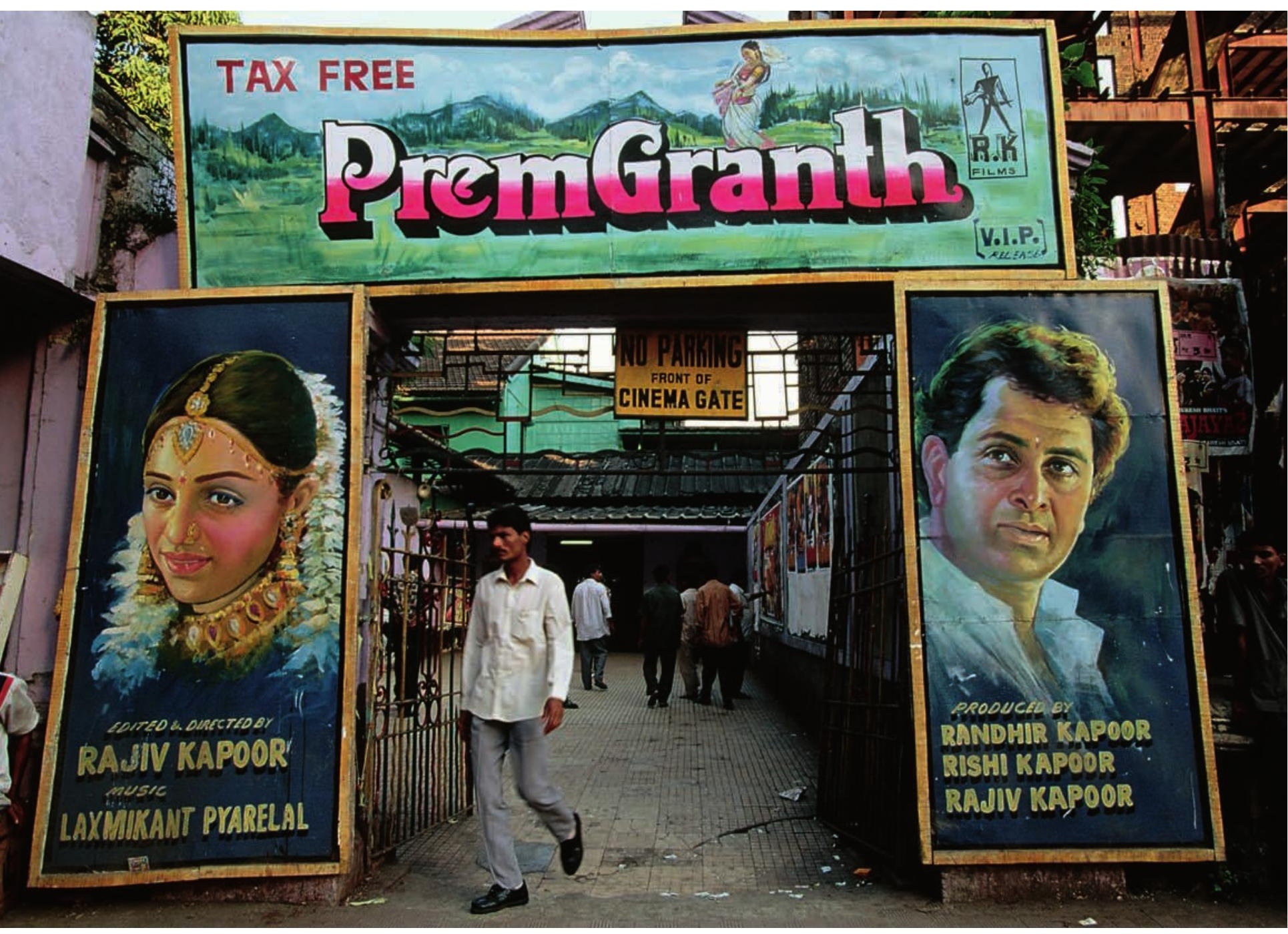

Fig. 2 Affiches de films, Bombay, 1996. Photo Catherine Karnow @ Catherine Karnow/Corbis.

originaux, difficilement reproductibles, voire quasi infalsifiables. Ce procédé est si répandu que certaines formations contemporaines s'en sont même fait une spécialité. C'est le cas des fanfares, ensembles accompagnant les fêtes, les mariages et les événements politiques, dont la singularité tient précisément au fait de jouer des musiques de tous horizons et toutes origines (Booth 1990, 2005). Il trouve enfin sa forme la plus aboutie dans les musiques produites par l'industrie cinématographique, souvent considérée comme capable de synthétiser tous les genres musicaux, qu'ils soient locaux ou internationaux ${ }^{6}$.

Si l'usage en est répandu, les termes qui désignent ce phénomène varient considérablement selon les contextes de production musicale. Tout d'abord, les mots «copie» ou "plagiat» sont majoritairement utilisés par les amateurs de musiques de films. Certains blogs et groupes sur Internet proposent par exemple d'échanger et de donner accès à des chansons qui s'appuient sur des mélodies d'emprunt. Je pense notamment au site web ItwoFS ("Inspirations in Indian Film Songs ». Music plagiarism. India. Oh my God!), qui recense actuellement
0

6. Sur les musiques de films dans le cinéma populaire hindi, voir notamment Arnold 1993, Booth 2008, Grimaud 2003, Manuel 1993. 
-

7. Ma traduction.

8. Par exemple, en hindi, les mots tarz ou dhun désignent la mélodie tandis que bol renvoie au verbe (Marcus 1995 : 101); ou encore, en malayalam, les mots rupam ou inam (correspondant à la mélodie) se distinguent de pattu', l'énoncé chanté (Guillebaud 2004 : 223-225; 2008 : 209-212]. Lusage du terme anglais tune est aussi largement répandu parmi les musiciens pour désigner les mélodies locales ou d'emprunt.

9. Sur les implications du remake d'un roman vers un script, voir l'analyse d'Emmanuel Grimaud à propos de La Nuit bengali de Mircea Eliade adaptée dans le film Hum dil de chuke sanam (2003: 78 et sq.).

10. Les plus grands studios de cinéma sont implantés dans les capitales de Bombay (en langue hindi), Madras (en langue tamoule) et Hyderabad (en langue télougou).

11. Celle-ci a estimé que «les lecteurs des romans de J.K. Rowling sont assez éduqués pour faire la différence entre Harry Potter et Hari Puttar, et comprendront sans difficulté qu'il ne s'agit pas du même héros » (BBC News, 22 septembre 2008). plusieurs centaines d'emprunts : entre des films produits à différentes époques, d'un cinéma régional à l'autre, de la pop américaine à Bollywood ou de musiques régionales au cinéma. L'auteur du blog écrit notamment :

L'objectif est direct et simple - fournir une liste d'inspirations musicales (parfois subtiles et intelligentes mais pour la plupart flagrantes!) des compositeurs de musiques de films indiens. L'autre point clé est la différence entre le fait de s'inspirer pour créer la mélodie et celui d'utiliser des basses/frappes/boucles rythmiques provenant de sources extérieures. Ces chansons dont la mélodie de base s'est inspirée sont précédées de l'abréviation $\langle\mathrm{TC}\rangle$, qui indique la copie d'une mélodie [tune copy]. Lorsqu'elles n'ont aucune abréviation, il s'agit del'utilisation de boucles rythmiques, frappes/basses, etc.?

L'idée du projet est bien de recenser les emprunts tout en offrant un accès direct aux enregistrements; toutes les sources sont en effet téléchargeables gratuitement. La base ainsi créée fonctionne sur le simple plaisir qu'il y a pour tout amateur à reconnaître des emprunts (ou transferts) mélodiques et à analyser les ressorts musicaux de la copie. L'usage de ce terme ainsi que celui de «plagiat » est ici moins négatif qu'il n'y paraît : il souligne un procédé de composition que ces amateurs se plaisent à dénicher, décrypter et échanger.

Le deuxième terme, "parodie" - traduction française de parody-, n'a en anglais aucune connotation péjorative ni satirique, mais désigne simplement le fait d'arranger un nouveau texte sur une mélodie d'emprunt (Manuel 1993 : 131). Il se rapporte plus généralement aux traditions de la no melodic composition (Marcus 1993 : 102 ; 1995) consistant à créer de nouveaux énoncés verbaux en s'appuyant exclusivement sur des mélodies préexistantes. Le terme, principalement utilisé par les musicologues anglophones, quelle que soit leur spécialité, n'a pas d'équivalent strict dans les langues indiennes, mais distingue de la même manière les paroles de la mélodie ${ }^{8}$. Il renvoie enfin aux enregistrements commerciaux appelés "versions », marché séparé de l'industrie du film et sans aucun équivalent dans le monde en nombre d'albums. Il s'agit de réinterprétations de chansons précédemment commercialisées, comme les hits de cinéma en langue hindi - recomposés dans différentes langues régionales (bengali, punjabi, marathi) -, les hits américains adaptés localement et enfin, à partir des années 1980, les interprétations en anglais de chansons pop hindi (Manuel 1993 : 145-150).

Enfin, le terme remake est presque exclusivement associé au monde du cinéma. À Bollywood, on peut faire le remake d'une histoire, d'un roman, d'une épopée, etc. ${ }^{9}$ Il concerne en outre de nombreux films anciens remis au goût du jour, ceux produits dans une région pour être « refaits » dans une autre ${ }^{10}$ ou encore les nombreuses versions de films hollywoodiens. On pense ici aux classiques comme Tarzan, Superman, Spiderman ou encore E.T., sans oublier les blockbusters les plus récents. En septembre 2008, le film Hari Puttar (littéralement «fils de dieu ") se présentait comme un remake de la production américaine Maman, j'ai raté l'avion sous un titre d'emprunt peu anodin! De manière inédite dans l'histoire du cinéma en Inde, il a fait l'objet d'une plainte judiciaire engagée par la Warner Bros, finalement déboutée par la haute cour de Delhi ${ }^{11}$. Ce type de procès est relativement récent et révèle la crainte du préjudice financier, d'ailleurs bien réel, suscitée aujourd'hui par l'internationalisation de la diffusion du cinéma populaire hindi et ses parts de marché grandissantes au Maghreb, au Moyen-Orient, en Asie du Sud-Est, en Afrique, en Europe et en Amérique du Nord.

Le remake s'applique tout autant à la chanson de film; il recouvre alors strictement le sens d'emprunt et de filiation qu'il revêt pour les amateurs dont nous avons parlé précédemment. 


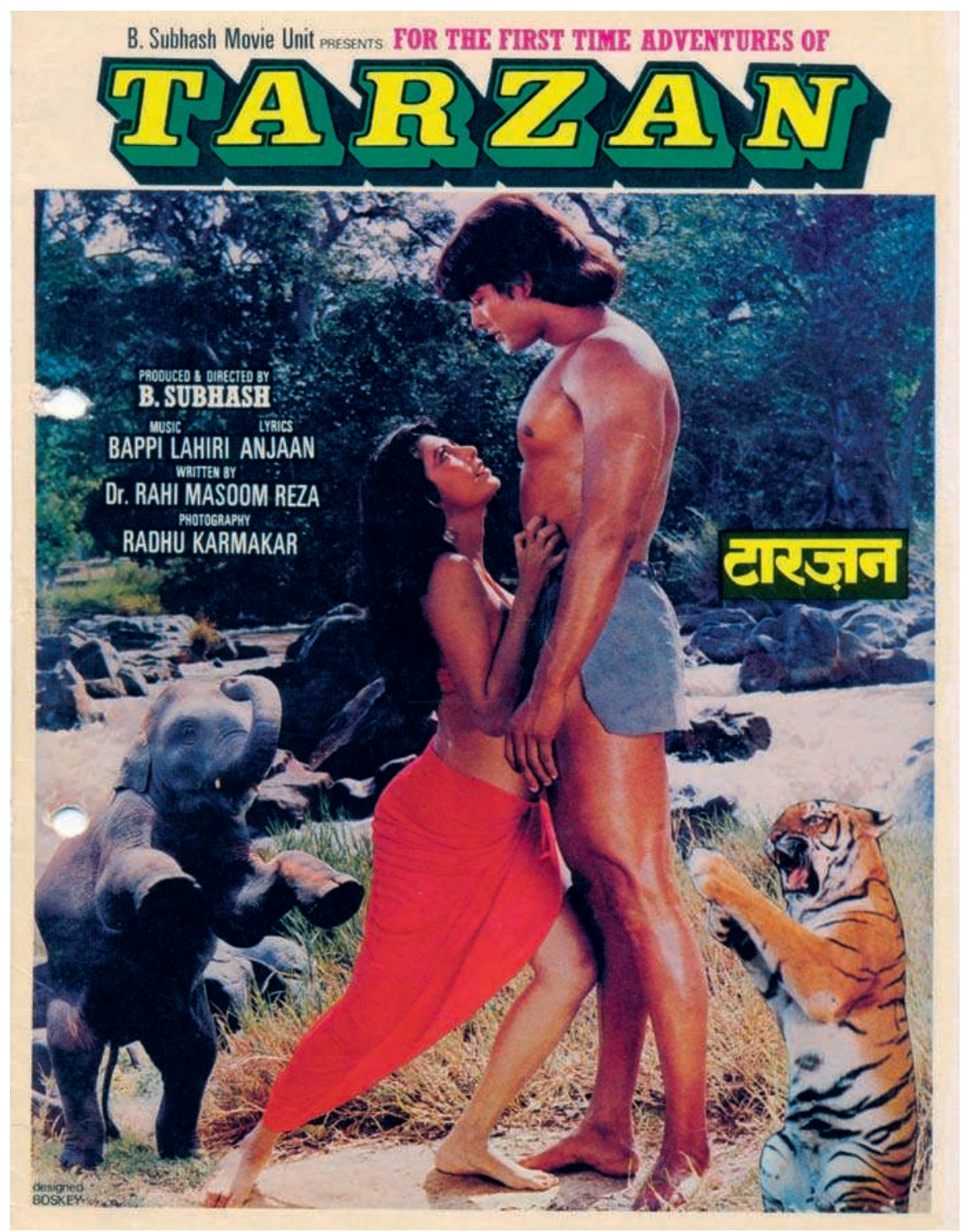

Fig. 3 Remake de Tarzan par Bollywood, réalisé par Babbar Subhash, avec Raja Duggal, Kimi Katkar et Hemant Birje, 1985. Droits réservés.

Les termes du débat ainsi définis, nous constatons qu'ils désignent tous la nature d'une même opération créative, à savoir l'emprunt d'un matériau préexistant tel qu'il s'applique à la chanson Nimbuda. D'abord chanson «locale» rajasthani, celle-ci devient ensuite chanson de film, une filiation chronologique attestée d'ailleurs par le blog ItwoFS où elle apparaît parmi une centaine d'autres exemples d'emprunts à la variété américaine, au jazz ou encore aux symphonies classiques occidentales. Pour les musicologues anglophones, la chanson se présente aussi potentiellement comme un cas typique de parody ou encore, dans les milieux du cinéma, comme un remake finalement assez ordinaire. Pourtant, comme on va le voir, la "carrière » de cette chanson se prête à des divergences d'interprétation très marquées, certes musicales mais pas seulement si l'on considère les discours qui circulent à son sujet dans le domaine public. 


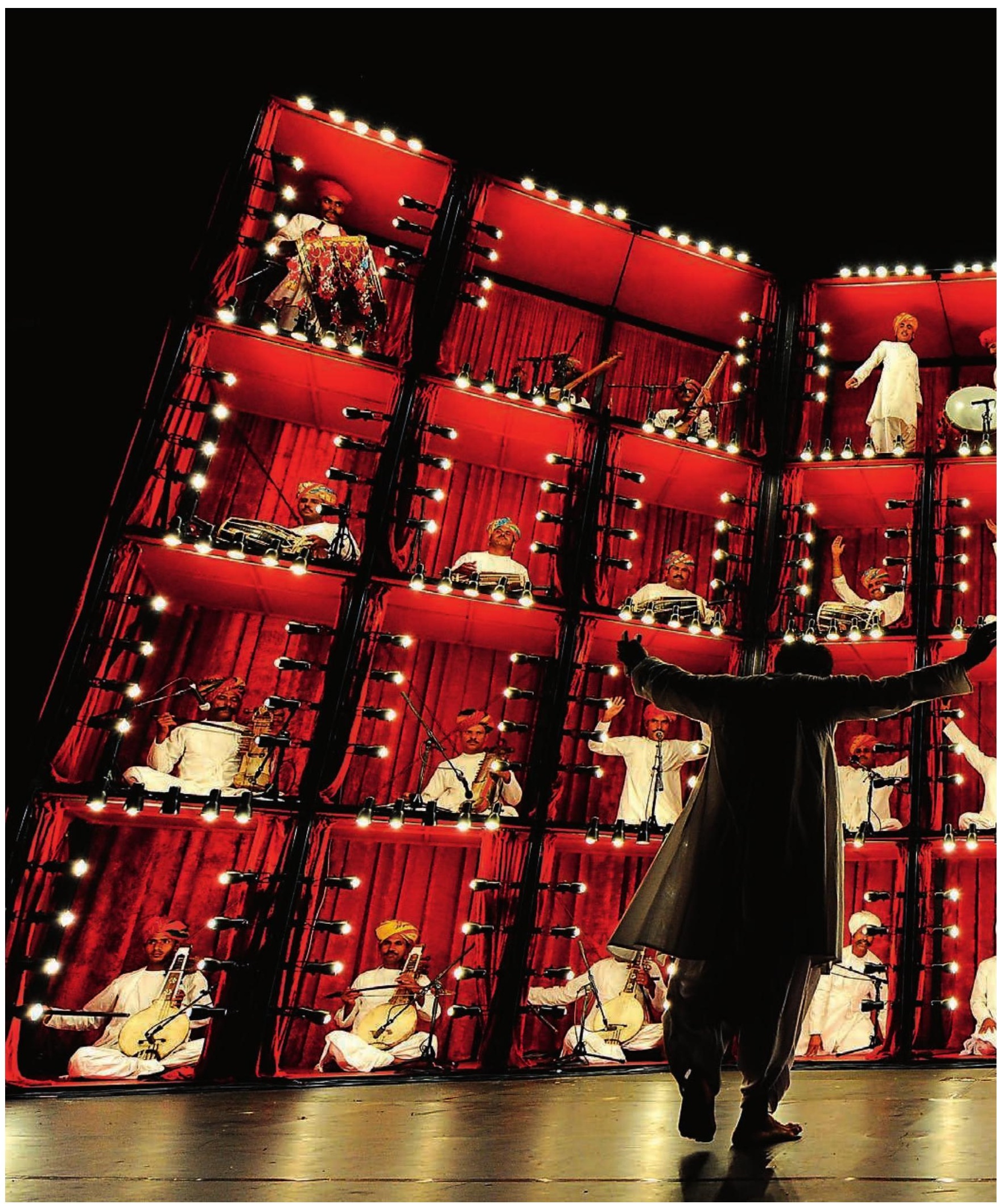

Fig. 4 Spectacle de musiciens du Rajasthan, The Manganiyar Seduction, au Sydney Festival en janvier 2010. Photo Torsten Blackwood (c) Torsten Blackwood/AFP. 


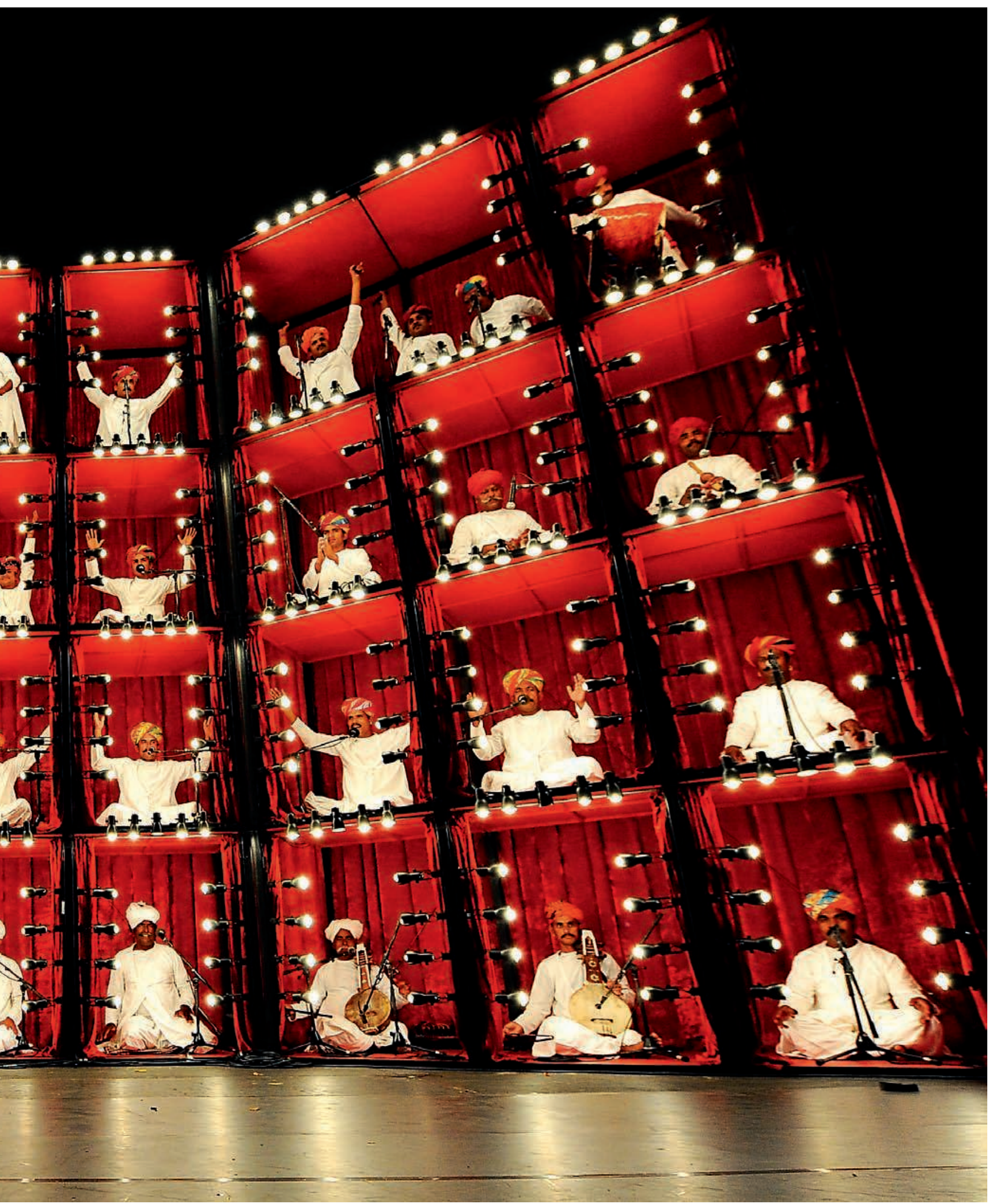


-

12. Les cas similaires d'emprunt sont courants en Inde, en particulier au Kerala, État du Sud où j'effectue mes recherches de terrain. Ils ne sont donc pas spécifiques à la caste des manganiyar. Cependant, aucun n'a à ce jour fait l'objet d'une controverse comparable à celle de Nimbuda.

13. Je m'appuie sur une première version publiée sur CD dans le cadre d'un hommage à ce chercheur (Bharucha 2003), livre d'entretiens recueillis auprès de l'intéressé avant son décès en 2004. La pièce y est décrite comme étant inspirée du padla, cérémonie préliminaire au mariage où la famille du futur mari envoie différents dons.

14. Sa publication sur CD en 2003 mentionne cependant directement le «compositeur » [A], qui aurait hérité la chanson de sa mère; ce type de chant associé au mariage est plus généralement chanté par les femmes.

15. Parmi les musiques régionales de l'Inde, ce répertoire est l'un des plus connus, diffusé aujourd'hui par les travaux académiques, les productions discographiques mais aussi plus récemment dans les festivals de musiques du monde. J'aurai l'occasion de revenir sur ce point.

16. Sur les implications culturelles, économiques et politiques du concours, voir notamment Assayag 1999.

17. Les compositeurs sont généralement désignés par l'expression music director.

\section{Citron amer : chronologie d'un processus d'emprunt}

Attribuée au chanteur rajasthani [A], spécialiste de caste manganiyar, la chanson est une allégorie du mariage, un thème par ailleurs commun à nombre de musiques régionales. Elle traite cette relation de manière poétique, à la fois douce et amère. J'ai pu écouter cette chanson par l'intermédiaire d'enregistrements et lors de prestations scéniques données par les musiciens de cette caste, en Inde et en France $^{12}$. Retracer la chronologie est ici important pour comprendre l'enchaînement des événements et le type de discours qui s'y attache successivement.

La première version a été enregistrée et déposée dans les archives du chercheur Komal Kothari ${ }^{13}$, célèbre ethnomusicologue fondateur d'un institut de recherche à Jaipur et auteur de différentes monographies sur les musiques du Rajasthan. Dans ce contexte académique et patrimonial, un tel enregistrement de terrain visait à documenter les performances de musiciens locaux. La chanson y apparaît donc pour ce qu'elle est au moment de la captation, à savoir une pièce appartenant au répertoire d'une caste particulière ${ }^{14}$.

EXTRAIT 1

Cet extrait et les suivants sont consultables sur http://gradhiva.revues. org/1814

Dans cette version, la chanson Nimbuda se présente dans une formation intime, une vièle kamaycha, habituellement jouée par ces spécialistes, accompagnant une voix d'homme soliste. Pour l'auditeur familier de ce répertoire ${ }^{15}$, la vocalité, les ornementations et mélismes ainsi que l'utilisation d'un rythme non mesuré font de cette chanson une production «localisée », c'est-à-dire portant les traits esthétiques signés de son spécialiste de caste.

L'année 1999 marque un premier tournant avec la sortie du film hindi Hum dil de chuke sanam ou HDDCS ("J'ai déjà donné mon cœur mon amour ") du réalisateur Sanjay Bhansali. Importante à plusieurs titres, cette production est d'abord un immense succès en Inde et inaugure la carrière de sa jeune héroïne, l'actrice Aishwarya Rai, tenante du titre prestigieux de miss Monde ${ }^{16}$ et dont la carrière cinématographique a depuis pris son essor au point d'en faire aujourd'hui l'actrice indienne la plus internationalisée. Outre ce casting, le film présente aussi plusieurs chansons signées du compositeur $[\mathrm{B}]^{17}$ qui ont connu un succès tout aussi retentissant. Comme dans bien d'autres productions à Bollywood, ces titres font un usage important de l'emprunt. Parmi les différentes séquences musicales, on peut reconnaître aisément le thème du célèbre Love Story américain ou encore la chanson manganiyar Nimbuda. À cet égard donc, le film ne se distingue pas véritablement des autres productions du genre.

\section{EXTRAIT 2}

Dans la version filmi, la chanson Nimbuda est entièrement identifiable par ses traits mélodiques et ses paroles (couplet/refrain), qui reprennent strictement les vers du chant manganiyar et ses onomatopées caractéristiques. Pour qui écoute cette version en contexte, c'est-à-dire filmée et dansée sur un plateau de tournage, l'expérience esthétique s'en trouve cependant radicalement transformée. L'actrice Aishwarya Rai, vêtue du costume traditionnel rajasthani, entre en scène par les marches d'un escalier, puis, sur une chorégraphie de type cabaret, se dirige jusqu'à la pièce centrale de la maison, la piste de danse. Le 


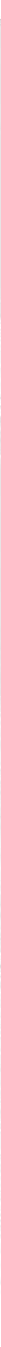

Fig. 5 Affiches de films tamouls. Pondichéry, 2003. Photo Amit Bhargava @ Amit Bhargava/Corbis.

spectateur peut facilement reconnaitre le style architectural de la haveli, maison traditionnelle de l'ouest de l'Inde. Il s'agissait dans cette scène "de faire folk» (Grimaud 2003 : 198) en mobilisant différents costumes, décors et accessoires propres à situer la scène dans une localité régionale typiquement rajasthani. Ainsi, la chanson et la danse visaient moins à se référer explicitement aux manganiyar qu'à créer pour les besoins du film une atmosphère culturelle type. Le thème du Citron amer choisi par le compositeur semblait également bien adapté à la narration du film; la chanson démarre en effet dans la seconde qui suit une dispute entre les deux amants. L'héroïne restée à l'extérieur de la pièce tente de se faire pardonner (une gifle partie trop rapidement), tandis que le héros lui montre son mécontentement en jetant un objet contre la porte, qu'il laissera définitivement fermée...

Dans la chanson Nimbuda, qui surgit à ce moment, l'on constate tout le travail de recomposition musicale effectué. Une relation particulièrement dynamique se crée entre le rythme musical et les changements de plans de caméra; chorégraphie et montage fonctionnent en une même unité d'expression. Sur la bande son, la chanson se distingue radicalement de sa première version par l'affluence 
des sources instrumentales et vocales mobilisées et leur orchestration. On peut notamment identifier certains instruments locaux, comme les claquettes de bois kartal, mais sans qu'aucune référence à des musiciens particuliers soit recherchée : il s'agit surtout de recréer l'ambiance d'une région, une sorte de «carte postale sonore ». Dans cette version composée en studio, d'autres éléments esthétiques tranchent fondamentalement avec l'esthétique manganiyar : la voix soliste est celle d'une femme (la célèbre chanteuse hindi Kavitha) et alterne avec un chœur d'hommes donnant une réplique énergique sur le refrain "nimbuda!»; le grain est sirupeux, le registre suraigu, le rythme entièrement mesuré; le tempo est très soutenu, la réverbération de règle, etc. Autrement dit, la marque de fabrique est bel et bien de style filmi et n'a pour ainsi dire plus rien de manganiyar, hormis bien sûr la mélodie d'emprunt.

Suite au succès du film, la chanson s'est diffusée dans tout le pays, faisant de Citron amer la chanson «du film HDDCS ». Parallèlement, le musicien manganiyar [A], et plus généralement les spécialistes de sa caste et même de sa région, n'ont cessé de l'interpréter. Il semble même que le succès du film ait eu des répercussions directes sur leur manière de l'exécuter, voire de se positionner en tant que professionnels de la musique. C'est en 2001, lors d'une représentation dans une grande salle parisienne, que j'ai pu en entendre une troisième version. Elle fut interprétée par un groupe de jeunes musiciens de caste du nom de Chota Divana, formé dans le cadre de différents festivals de musiques du monde en France et en Europe ${ }^{18}$. Force est de constater que l'effet du film ne se fit pas attendre chez ces chanteurs. En termes musicaux tout d'abord, puisque la forme couplet/refrain développée dans le film a été adoptée telle quelle dans leur interprétation.

\section{EXTRAIT 3}

Ày regarder de plus près, il apparaît que seuls certains traits de la version filmi ont été clairement incorporés : l'introduction chantée était réduite à son développement minimal et le refrain - répété plusieurs fois de suite à la manière bollywoodienne - devenait le support principal de la chanson. Seul le style vocal des chanteurs ne semblait en rien calqué sur celui de la chanteuse de studio. À cet égard, les manganiyar, pourrait-on dire, restaient bien des manganiyar, à savoir des interprètes ayant développé leur propre marque de fabrique.

De manière plus générale, il semble que les musiciens ont naturellement tourné à leur avantage les fruits du succès obtenu par le film. Comme bien d'autres artistes promus sur les scènes internationales, ils n'hésitent pas aujourd'hui à se présenter comme des interprètes "légitimes" et "ancestraux». Si leur interprétation du Nimbuda «original» devient aujourd'hui si importante dans leur discours, c'est aussi parce qu'elle sert leurs intérêts, à savoir être programmés dans les festivals. De même, cette nouvelle popularité acquise indirectement par le film contribue à revaloriser leur statut dans une société locale qui, traditionnellement, leur assigne une position de

- -

18. Ce groupe a la particularité d'être composé de solistes enfants. Il a été précisément constitué pour des tournées en Europe.
Fig. 6 Danseurs s'entraînant pour une séquence de Chor Machaye Sohr aux Filmalaya Studios, Bombay, 200o. Photo Jonathan Torgovnik @ Jonathan Torgovnik/Getty Images.

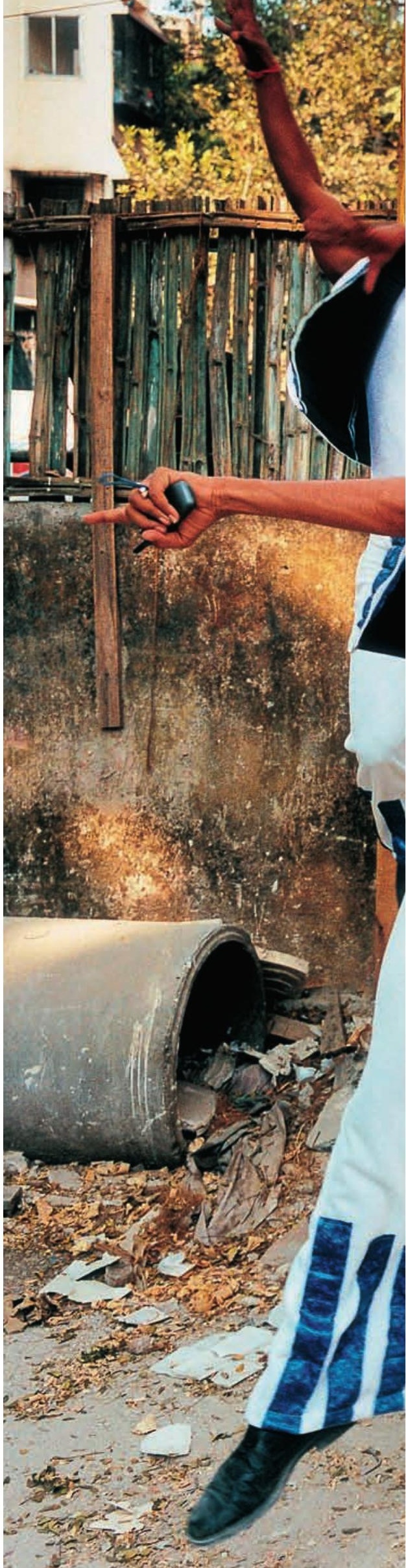




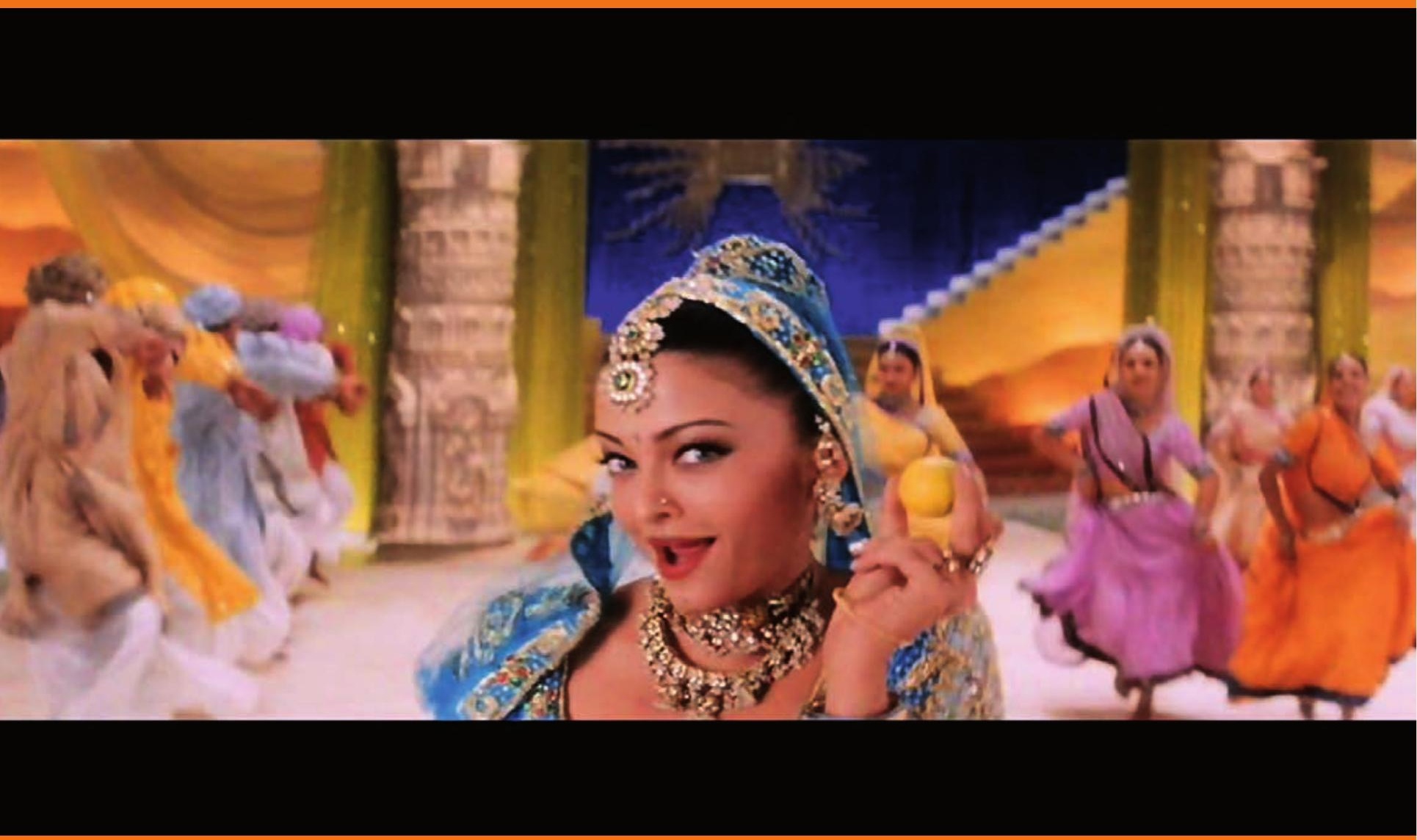


dominés, celle du spécialiste de basse caste. Bien avant la sortie du film, les musiciens professionnels, tels les manganiyar, avaient déjà connu des transformations importantes de leurs réseaux de commanditaires. Autrefois liée à la vie musicale des cours princières, leur activité de caste s'est aussi adaptée à de nouveaux contextes, celui des politiques culturelles étatiques et du mécénat privé, au cœur de l'efflorescence des festivals en Inde et à l'étranger. Le succès de la chanson Nimbuda vient donc renforcer un réseau de diffusion déjà consolidé. Il les propulse aussi dans le milieu de la jet set bombayite et élargit leur notoriété individuelle dans la presse ${ }^{19}$. Notons ici que le film semble avoir servi de «faire-valoir » supplémentaire pour ces musiciens, ce que confirme aussi l'écoute comparée des trois enregistrements que nous avons esquissée. En empruntant certains traits esthétiques à un succès médiatique, à la manière d'un label "vu au cinéma ", les musiciens s'assurent en effet les faveurs d'un public élargi. Que le public connaisse ou non le film, le fait de programmer la chanson lors des prestations scéniques et des festivals n'est pas anodin. Celle-ci devient ainsi un emblème de leur répertoire de caste, une manière de se présenter à un auditoire, même si le public étranger (c'est le cas de la salle parisienne dont nous avons parlé) ne peut toujours véritablement saisir l'épisode d'emprunt.

\section{Au fil des argumentations}

Ces emprunts très fréquents entre cinéma et musiques régionales (et vice-versa) suivent à chaque fois une séquence chronologique particulière. Dans le cas de Nimbuda, le sens de l'emprunt est parfaitement identifiable : du musicien rajasthani au compositeur de musiques de films, puis du film vers le musicien local. Ce parcours n'est en rien anodin, car c'est bien l'ordre de cet enchaînement qui est à la source des divergences d'interprétations, allant du simple jugement esthétique à la condamnation systématique de l'emprunt, ou inversement à sa très grande valorisation arguant de son bien-fondé culturel en termes de créativité musicale.

Lorsque les musiciens empruntent des mélodies au cinéma, les musicologues les plus traditionalistes diront qu'il n'est pas bon que les chanteurs importent en même temps les images associées à la chanson dans le film. S'ils les intègrent dans un acte rituel ou dévotionnel, par exemple, l'emprunt est le plus souvent condamné par nombre d'intellectuels locaux. Par leur double rôle de chercheurs et de promoteurs des musiques (Guillebaud 2010 sp.), certains se positionnent en gardiens des normes «traditionnelle ${ }^{20}$ ». Autre argument courant : les musiciens locaux seraient victimes d'une imposition médiatique qui limiterait leur créativité et les forcerait à emprunter en dehors de leur culture d'appartenance. Dans les deux cas, le genre filmi est perçu négativement comme un genre hybride, pas assez indien et peu sérieux - en témoigne l'expression courante et péjorative, this is totally filmi!, utilisée par ces intellectuels.

Du côté des musiciens, au contraire, la question de l'origine d'une mélodie est généralement peu pertinente. Ils répondent souvent de manière détachée : «C'est juste une mélodie, l'histoire reste la même! » Le musicien perçoit donc davantage le procédé créatif d'emprunt lui-même, voire en estompe la filiation, sans toujours prêter attention à ses implications en terme d'identité culturelle. Les airs sont ainsi dissociés de leur contexte, suivant un processus de creative resignification (Manuel 1993: 140). Les mélodies auraient leur vie propre en dehors de leur source d'origine. Il est d'ailleurs intéressant de noter que les chercheurs
PAGE CI-CONTRE

Fig. 7 Photogramme du film Hum dil de chuke sanam (" J'ai déjà donné mon cœur mon amour "), réalisé par Sanjay Bhansali, avec Aishwarya Rai, 1999. Droits réservés.

DOUBLE PAGE SUIVANTE

Fig. 8 Les acteurs Govinda et Sunali Bendre pendant le tournage du film Jis Desh Mein Ganga Rehta Hai ("Dans le pays où vit Ganga »), 2000, Mahabaleshwar. Photo Jonathan Torgovnik @ Photo Jonathan Torgovnik/Getty Images.

\footnotetext{
19. Depuis dix ans, plusieurs articles de presse ont été consacrés au chanteur $[\mathrm{A}]$ et, récemment, celui-ci a même été invité au mariage de l'héroïne du film, Aishwarya Rai, avec l'acteur Abhishek Bachan. À propos du couple de stars, [A] déclare d'ailleurs, non sans humour : «S'ils nous invitent, nous sommes plus qu'heureux d'y aller. Nous chanterons le Nimbuda original et nous leur donnerons nos bénédictions! » (CNN-IBN, 15 avril 2007) Ce discours détonne plutôt avec celui émanant des intellectuels locaux, notamment, comme nous le verrons plus loin.

20. Cet argument d'authenticité se rencontre aussi fréquemment au sein des jurys d'audition de la All India Radio, grand mécène national qui fournit des contrats d'enregistrement aux musiciens (Guillebaud 2008 : 129-130).
} 


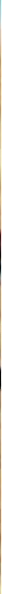

-1 A

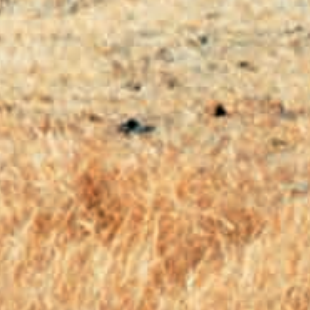

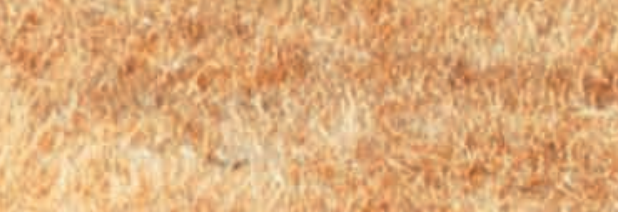

How

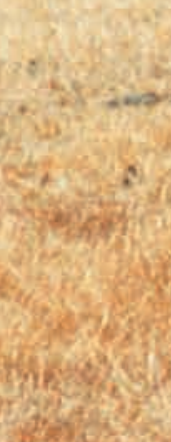

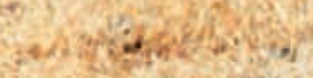

ofese
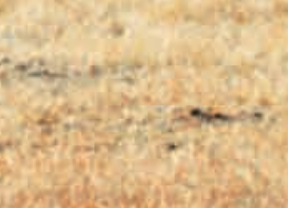

Mosecosy

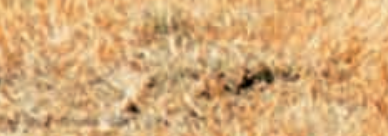

(a) soln

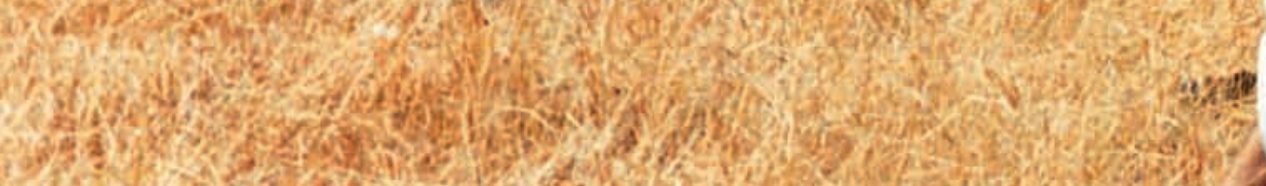
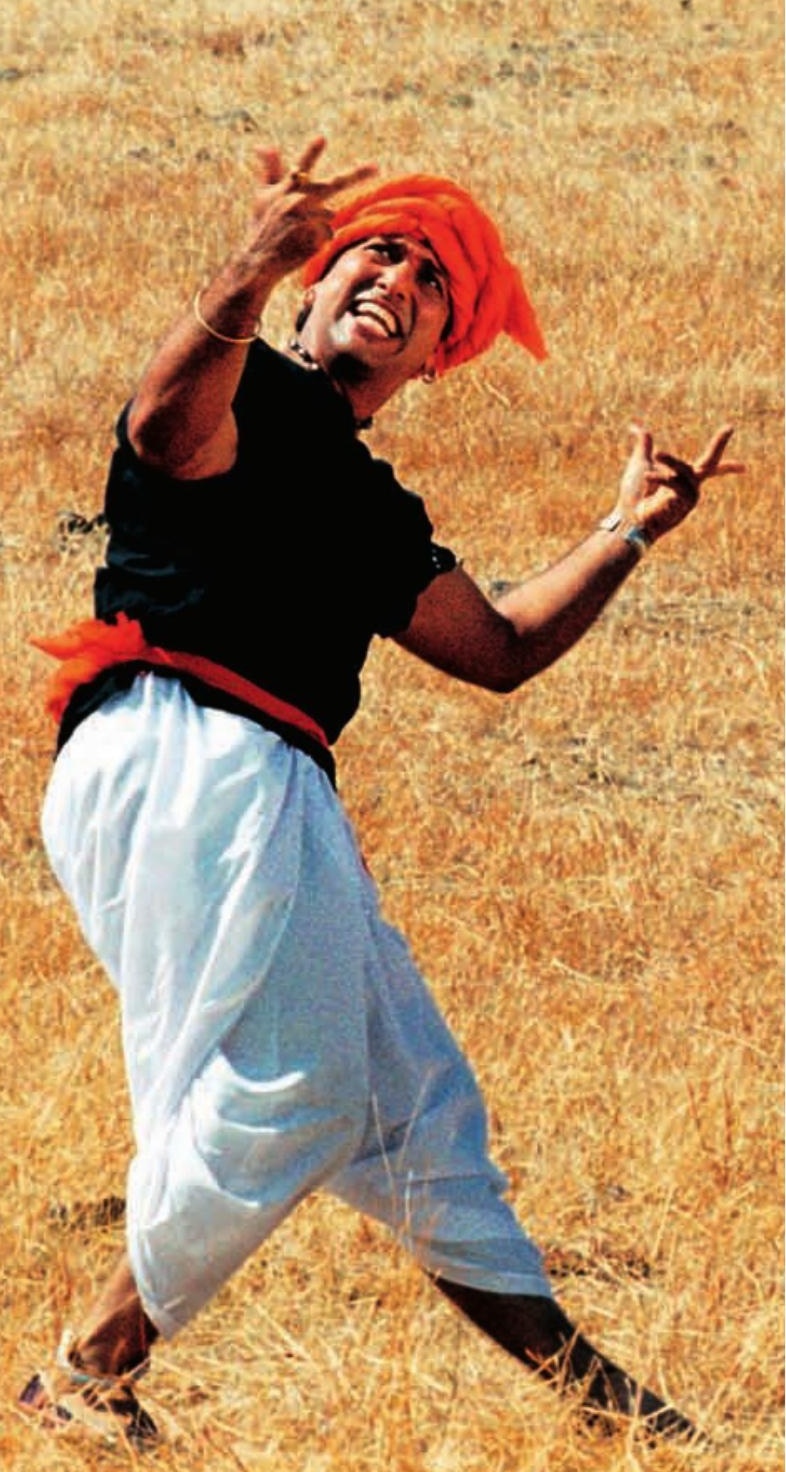


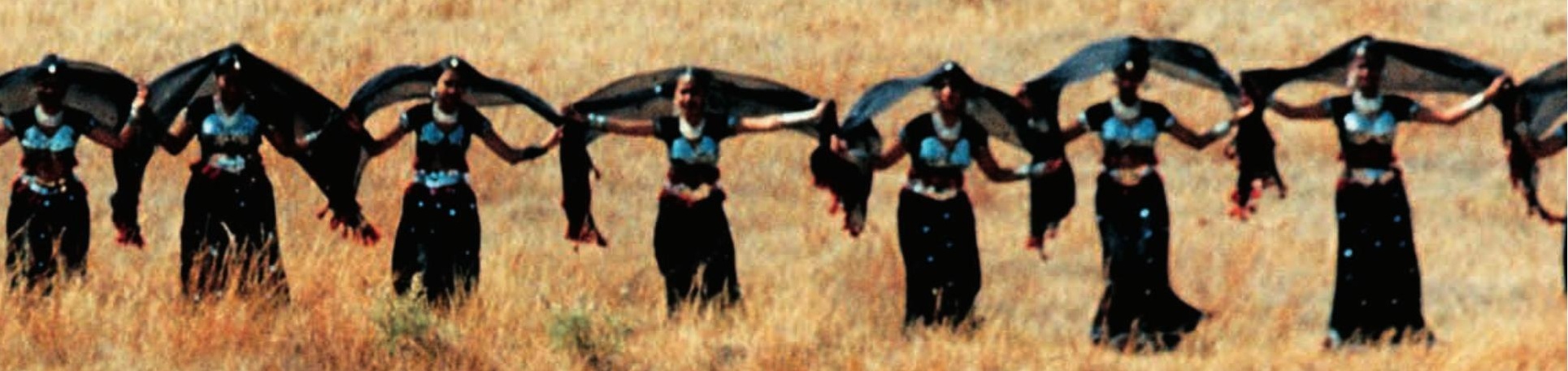

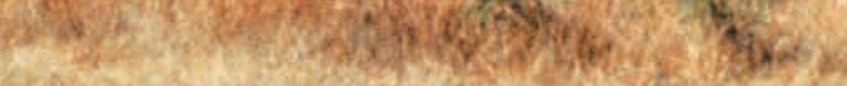

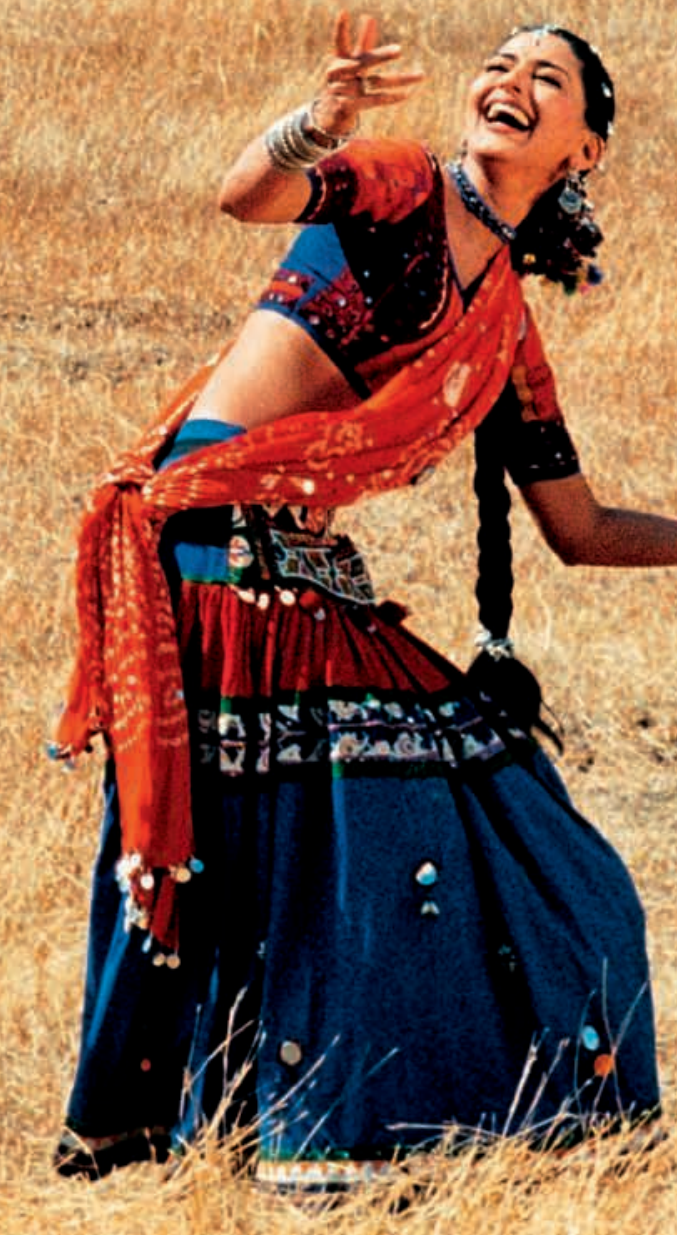

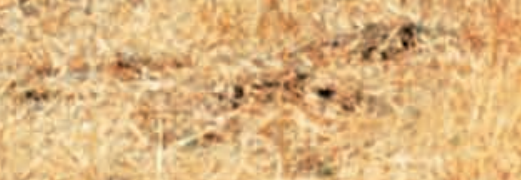

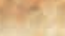

$$
\text { . }
$$


(nord-américains cette fois) ayant souligné de tels emprunts en donnent une analyse dans des termes différents, centrée cette fois sur le musicien. Sa capacité à intégrer ces sources est par exemple valorisée comme une manière pour lui de se définir en tant qu'individu «local » face aux phénomènes de globalisation. Le fait d'assimiler une mélodie de film, de renouveler la tradition et de se positionner par rapport aux médias tout en gardant son identité régionale est souvent mis en avant dans les analyses (voir par exemple Marcus 1993 : 105, 108). Cellesci peuvent cependant paraître réductrices dans la mesure où elles ne se fondent pas toujours sur une approche détaillée du procédé d'emprunt lui-même. Pour les musiciens, le fait de réutiliser une mélodie n'est pas toujours porteur d'une intention ou d'une stratégie; les notions d'«identité " et de « modernité » étant en réalité souvent mobilisées par les chercheurs eux-mêmes (sans que l'on sache au fond le sens qu'elles revêtent en musique)... Laissons donc aux musiciens le loisir d'agir aussi par simple goût pour un air!

Dans le monde du cinéma, l'argumentation est encore différente. Les compositeurs empruntent des mélodies locales (mais pas seulement) en leur donnant une nouvelle traduction en hindi ou en modifiant les paroles. Comme dans le cas de Nimbuda, ils contribuent également à créer une nouvelle esthétique, tout en s'inscrivant souvent dans des genres comme le rock, le disco, le hip-hop, etc. Le nombre d'emprunts est faramineux et ils concernent tout autant les musiques régionales que classiques (indiennes et occidentales), les hits européens, américains, ou encore la pop arabe. Lorsqu'ils évoquent cette pléthore de mélodies empruntées, les critiques de cinéma et les journalistes tentent aussi d'en expliquer la cause : ils soutiennent par exemple que, si les compositeurs empruntent tant, c'est qu'ils subissent la pression de leur producteur afin de produire toujours plus de chartbuster hits. Lors d'une émission télévisée récente sur le thème «Bollywood copying tunes. Inspiration or theft? ", un intervenant du plateau prit par exemple position en faveur des compositeurs de films indiens en général. Il affirmait qu'il ne fallait pas trop vite les accuser et qu'ils auraient même un

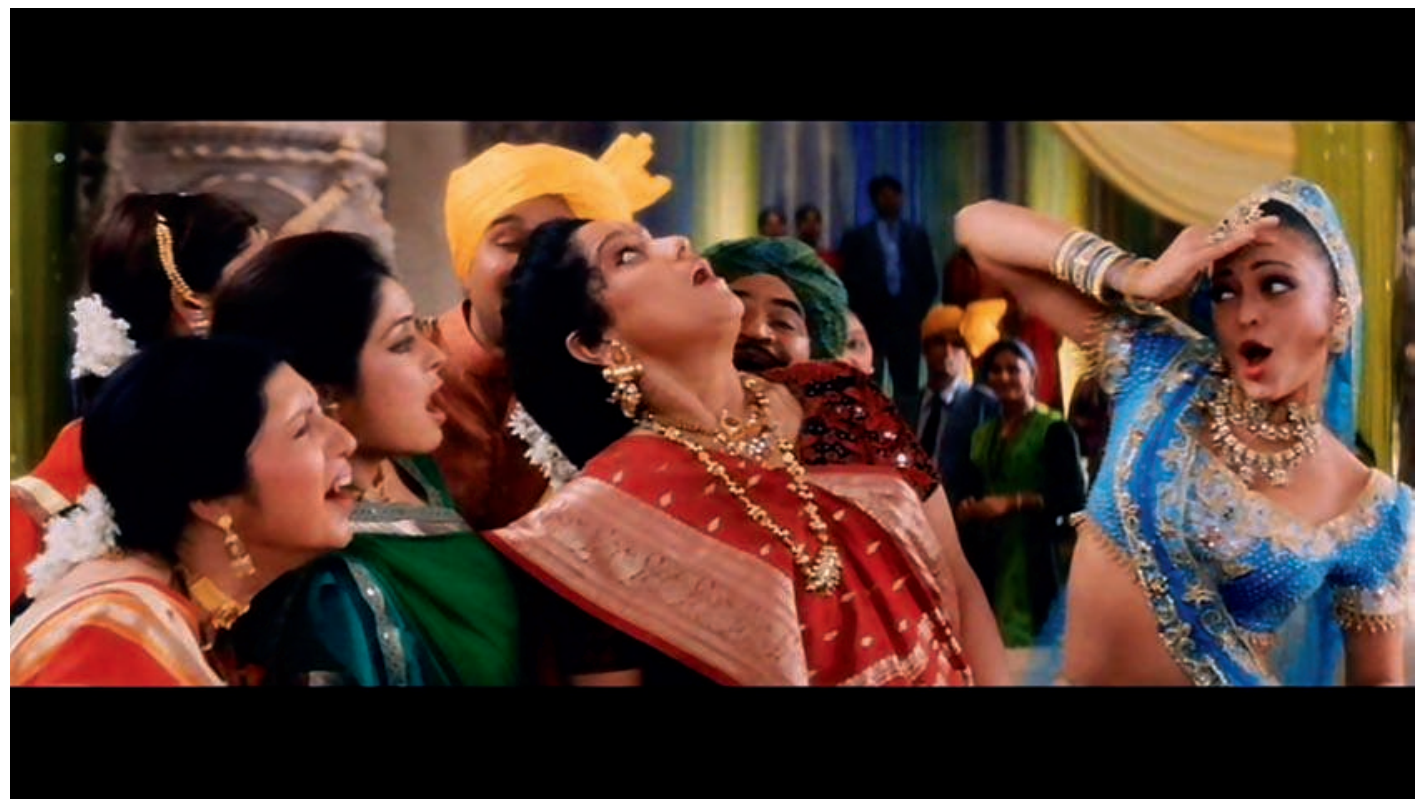

Fig. 9, 10 et 12 Photogrammes du film Hum dil de chuke sanam ("J’ai déjà donné mon cœur mon amour »), réalisé par Sanjay Bhansali, avec Aishwarya Rai, 1999. Droits réservés. 


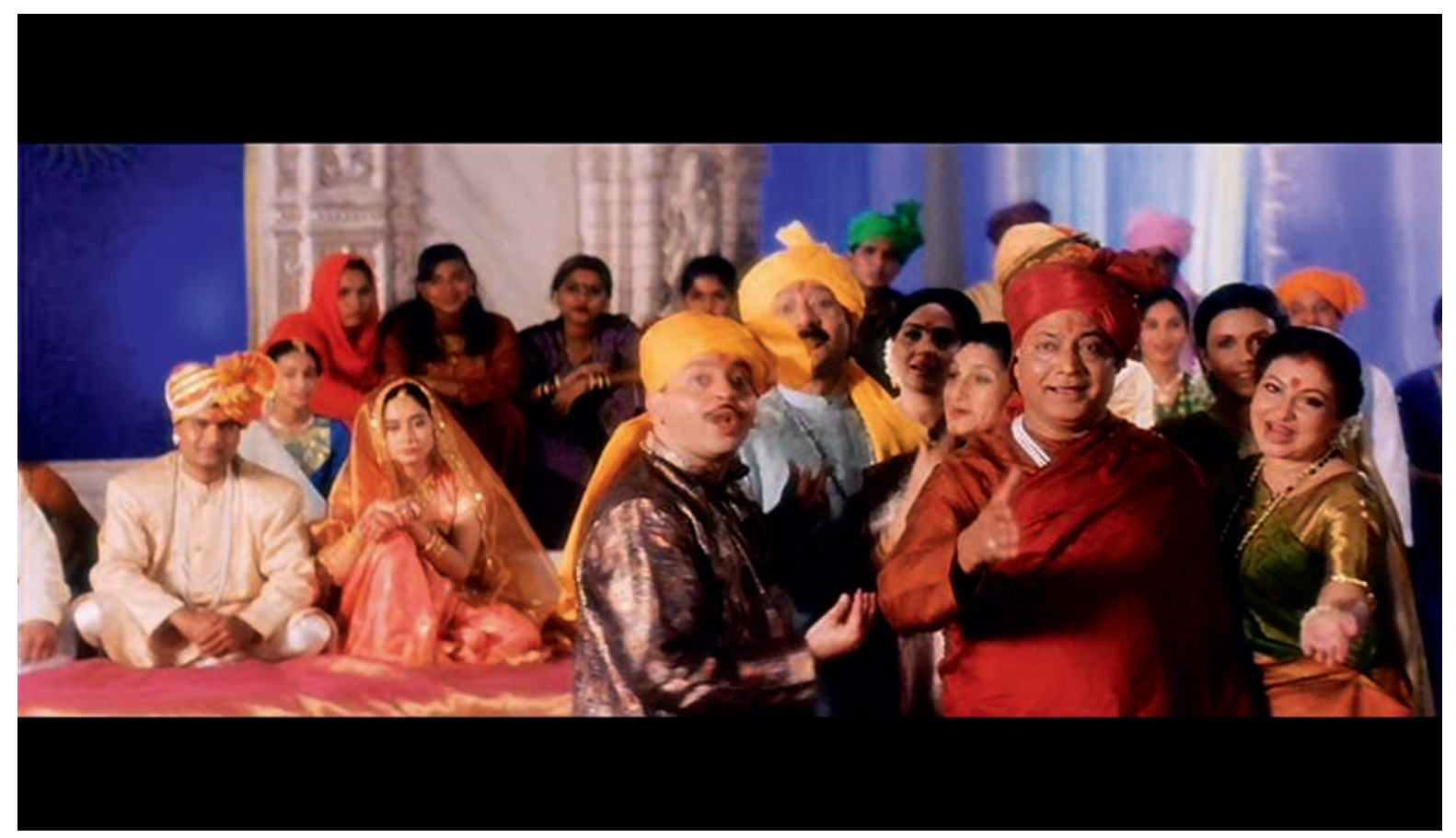

certain mérite à composer « près de six ou sept chansons par film et pour trente à cinquante films par an! Les compositeurs britanniques ou américains, eux, produisent au plus un album tous les cinq ou six ans [...]. Le blâme est donc plutôt à mettre du côté du directeur ou du producteur! »

De leur côté, les compositeurs de musiques de films n'adhèrent pas à ce discours. Ils expliquent en effet que, lorsqu'il y a des demandes de tune copying, "cela se fait toujours dans une discussion avec le réalisateur ou en fonction d'une scène ou d'un acteur particulier ». À cet égard, le célèbre compositeur Anu Malik se défend fermement de créer grâce à une inspiration extérieure ou un vol, qui lui seraient soi-disant imposés par la production. En direct sur le plateau de télévision, il dit : «Dites-moi, si vous me posez la question. Pensez-vous qu'un compositeur puisse survivre pendant vingt-cinq ans sans sa propre originalité ? Impossible! [...] Si les producteurs viennent vers moi, c'est pour mon propre talent. "

Force est de constater que ce type de discours touchant à l'originalité des musiques ou à un modèle de création typiquement "indien " émane presque exclusivement des acteurs liés au monde du cinéma et n'est finalement jamais tenu en dehors, notamment parmi les musicologues. Le travail spécifique des compositeurs de musiques de films est par ailleurs trop rarement analysé pour lui-même et souvent abordé sous le seul angle quantitatif du nombre d'emprunts effectués.

\section{Création versus éthique?}

Dans le cas précis de Nimbuda, la controverse naît surtout du fait que le sens de l'emprunt est économiquement dissymétrique : pour simplifier, le «riche » compositeur de Bombay s'empare de la mélodie du "pauvre » villageois. Et c'est précisément dans ce type de situation que l'argument d'infraction au droit d'auteur est toujours invoqué. Ainsi peut-on lire dans la presse à propos du chanteur [A] que son chant a tout simplement été « volé ». Au premier abord, le doute plane en

DOUBLE PAGE SUIVANTE

Fig. 11 Salle de montage aux Film City Studios à Bombay, 2007. Photo Jonathan Torgovnik (c) Jonathan Torgovnik/Getty Images. 
effet. Les compositeurs de musiques de films sont inscrits à l'Indian Performing Rights Society alors que les musiciens de village ne le sont pas. Pour Nimbuda, le chercheur Komal Kothari aurait pris position en ce sens afin de défendre les droits du chanteur auquel est attribuée la chanson. Il déclare à la presse :

La version originale rajasthani avait été enregistrée dans un album privé. Et maintenant, quelqu'un de Bombay vient, vole son chant et récolte tous les honneurs! [...] Les manganiyar ne pourront plus jamais interpréter leur chant. Ils doivent obtenir une autorisation et peut-être même payer des droits au compositeur de Bombay pour leur propre chant. C'est complètement injuste ${ }^{21}$. (The Sunday Tribune India, 21 janvier 2001)

En réalité, la mention relative aux droits d'auteur (qu'auraient à payer les musiciens) reste théorique et n'est de fait jamais appliquée; et ce, quelle que soit la musique mise sous copyright. Par ailleurs, l'infraction supposée concernant l'enregistrement privé paraît peu vraisemblable. La reprise filmi de la chanson s'appuie, on l'a vu, sur une nouvelle interprétation (et non des moindres d'un point de vue esthétique) et ne se fonde à aucun moment sur la reproduction stricto sensu du support enregistré lui-même.

Il ne s'agit pas ici de discuter de la dissymétrie économique entre les protagonistes, certes flagrante et indéniable, mais de souligner qu'elle ne suffit pas, à elle seule, à rendre compte de la complexité du processus d'emprunt dans sa chronologie spécifique. Comme l'exprime très justement l'ethnomusicologue Marc Perlman à propos d'une controverse sur le terrain indonésien voisin, «la rumeur [d'exploitation frauduleuse] peut être fausse mais vraie émotionnellement » (2009). Si le cas du chanteur [A] suscite sans aucun doute une certaine compassion, doit-on cependant réduire l'affaire Nimbuda à un simple « vol » au nom d'une criante inégalité sociale et économique? Certains auteurs réfléchissant au copyright dans les musiques de tradition orale ${ }^{22}$ verraient plutôt dans cet épisode :

Une plus grande prise de conscience des droits de propriété intellectuelle parmi les musiciens. Il y a eu une longue tradition de musiques de films qui utilisait les mélodies traditionnelles sans plus de réaction. D'un autre côté, il faut noter que les chanteurs populaires ont adapté durant de nombreuses années les mélodies de films et de chansons, qui sont effectivement sous copyright, dans leurs propres performances et ce, sans ramifications légales ${ }^{23}$. (Chaudhuri 2009 : 21)

Cette prise de conscience est aussi liée à la manière dont les chercheurs ont fait évoluer leur discours sur ces musiques, longtemps réputées "sans auteur" et généralement abordées sous leur seul aspect collectif et le plus souvent anonyme. Comme le confie aussi Kothari à propos du cas Nimbuda :

Cette situation nous fait prendre davantage conscience des «nouveaux compositeurs " parmi les musiciens - ces compositeurs tels Bhungar Khan et Ramzan Khan dont les noms sont reconnus par d'autres chanteurs populaires. Alors, il est évident qu'une sorte d'« auteur-ité " [authorship] est à l'œuvre ici, et que ces compositeurs ont des droits sur leurs propres compositions ${ }^{24}$. (in Bharucha 2003 : 279)

Le fait de décrire (et de dénoncer) l'emprunt sur le seul mode de l'infraction au

-

21. Ma traduction.

22. Voir notamment Seeger 1996 et la bibliographie indicative publiée in Seeger et Chaudhuri (éd.) 2004 : 291-292.

23. Ma traduction.

24. Ma traduction. droit d'auteur participe aussi d'un positionnement problématique de la part des musiciens. Certains disent par exemple publiquement : « Nous voulons garder nos arts traditionnels vivants, quel mal y a-t-il à ce que notre musique soit popularisée dans les films? " (The Sunday Tribune India, 21 janvier 2001) D'autres recherchent souvent une approbation extérieure par un «c'est plutôt bien, non ? ", lorsqu'on leur demande explicitement comment ils perçoivent la réutilisation de leur musique par le milieu du cinéma. D’autres encore argumentent dans le sens du vol et de l'in- 
fraction aux droits d'auteur qu'ils intègrent peu à peu à leur pratique. Cependant, on constatera que, même dans ce dernier cas, le jugement qualitatif de l'emprunt est généralement porté davantage par leurs mécènes (et médiateurs culturels) que par les intéressés eux-mêmes. Au mieux y verra-t-on une marque de leur « naïveté " face aux enjeux contemporains, comme l'analysent certains chercheurs... Un tel argument mérite cependant d'être reconsidéré à la faveur du musicien qui, du fait même de son statut culturellement dévalorisé, attire souvent la condescendance d'une certaine intelligentsia locale. La nuance s'impose donc. Si le fait de valoriser la créativité locale et ses figures traditionnelles semble justifié d'un point de vue éthique, il ne devrait pas masquer pour autant l'existence de ces figures plus médiatiques, ces chanteurs plus intégrés aux réseaux de diffusion nationaux et internationaux qui ont indirectement bénéficié du succès de la chanson.

Le cas Nimbuda ici esquissé révèle au grand jour la contradiction profonde que pose le droit d'auteur vu sous l'angle de l'emprunt de mélodies. Il montre que le point de vue juridique a la particularité d'émerger dans certains cas d'emprunt seulement, notamment en fonction de sa chronologie spécifique. Lorsque l'emprunt s'effectue du film vers le musicien local, personne n'est en effet enclin à le questionner en termes de propriété intellectuelle, mais plutôt sous l'angle de l'authenticité, de la créativité, ou même à ne pas le questionner du tout. Tout se passe comme si les mécanismes traditionnels de domination, de caste et de revenus économiques étaient mieux à même d'orienter les débats sur la propriété intellectuelle; ou, en caricaturant légèrement : plus le musicien serait riche (tel le compositeur de musiques de films), moins ses intérêts " propriétaires » auraient de sens à être débattus ou défendus... L'argument n'est pas sans rappeler le «mythe du pauvre auteur en lutte» sur lequel reposerait le droit d'auteur, particulièrement bien analysé par les juristes se plaçant dans la perspective du copyleft (Liang, Majumder et Suresh 2005). Il correspond à ce que Perlman nomme la « victimisation culturelle » (2009), notion qui semble faire marcher à plein les soupçons et présomptions d'infraction au copyright sur les terrains actuels des ethnomusicologues.

Aujourd'hui, dans les tribunaux indiens, les batailles de droit d'auteur concernent exclusivement les musiques de films, c'est-à-dire de compositeur à compositeur, alors que les procédés d'emprunt, on l'a vu, s'étendent sur des spectres bien plus larges. Cette situation confirme, si besoin était, que les questions de créativité musicale et de droit d'auteur sont loin de se recouvrir mutuellement. Elles ne concernent pas (du moins pas encore?) les rapports aux musiques régionales, aux hits américains ou encore à la pop arabe, dont les emprunts sont pourtant tout aussi fréquents ${ }^{25}$. Cette réalité s'explique aussi par le fait que les protagonistes impliqués dans le procédé d'emprunt - musiciens, compositeurs, chercheurs, législateurs - ne portent pas non plus au départ les mêmes projets. Cette liste semble d'ailleurs encore s'allonger aujourd'hui; au rythme de la carrière de la chanson Nimbuda, qui se poursuit dans d'autres répertoires locaux mais aussi dans celui des remix des hits hindi aujourd'hui très largement diffusés.
25. Le débat a cependant déjà eu lieu à propos de chansons pop américaines réutilisées par la jeune star coréenne G-Dragon, laissant inaugurer, à la manière d'Hari Puttar précédemment mentionné, de nouveaux cas jurisprudentiels. Ceux-ci témoignent surtout des manques à gagner des producteurs américains face aux industries culturelles émanant des nouvelles puissances mondiales.

CNRS- Université Paris Ouest-Nanterre

cguillebaud@free.fr

mots clés / keywords : Bollywood // Bollywood • musique // music • Rajasthan // Rajasthan • emprunt // borrowing - droits d'auteur // copyright. 


\section{Bibliographie}

\section{Arnold, Alison E.}

1993 «Aspects of production and consumption in the popular Hindi film song industry », Asian Music 24[1] : 122-136.

\section{AssaYAG, Jackie}

1999 «La "glocalisation" du beau. Miss Monde en Inde, $1996 »$, Terrain 32 : 67-82.

\section{BeAster-Jones, Jayson}

2010 «Evergreens to remixes: Hindi film songs and India's popular music heritage », Ethnomusicology 53(3) : 425-448.

BHARUCHA, Rustom

2003 Rajasthan. An Oral History: conversations with Komal Kothari. New Delhi, Penguin India.

\section{Bоoтн, Gregory D.}

1990 «Brass bands: tradition, change, and the mass-media in Indian wedding music », Ethnomusicology 34[2] : 245-262.

2005 Brass Baja. Stories from the world of Indian wedding bands. Oxford, Oxford University Press.

2008 Behind the curtain. Making music in Mumbai's film studios. Oxford, Oxford University Press.

BurKHOlder, Peter J.

1994 « The uses of existing music: musical borrowing as a field 》, Notes $50(3): 851-870$

1995 All Made of Tunes: Charles Ives and the uses of musical borrowing. New Haven-Londres, Yale University Press.

\section{CHAUdhuri, Shubha}

2009 Intellectual Property Management in a ethnomusicology archive. An empirical view from India. Genève, World Intellectual Property Organisation (WIPO).

\section{Giger, Andreas}

1994 «A bibliography on musical borrowing », Notes 50[3] : 871-874.

\section{GrimaUd, Emmanuel}

2003 Bollywood Film Studio ou comment les films se font à Bombay. Paris, CNRS Éditions.

\section{Guillebaud, Christine}

2004 « De la musique au dessin de sol et vice-versa. Un répertoire kéralais de formes sonores et graphiques », Cahiers de Musiques Traditionnelles 17 : Formes musicales, 217-240. itinérants du Kerala. Paris, CNRS Éditions

(«Sciences sociales du monde indien »; DVD-rom encarté].

2010 (sous-presse) « Music and politics in Kerala: Hindu nationalists versus marxists », in Daniela Berti, Nicolas Jaoul et Pralay Kanungo (éd.), The Cultural Entrenchment of Hindutva. Delhi, Routledge.

\section{KotHARI, Komal}

1972 Monograph on Langas: a folk musician caste of Rajasthan. Borunda-Jodhpur, Rajasthan Institute of Folklore.

1973 «The Langas. A Folk-Musician Caste of Rajasthan », Sangeet Natak 27 : 5-26.

1990 «Patronage and Performance », in Narendra Kumar Singh et Rajendra Joshi (éd.), Folk, Faith and Feudalism. Jaipur-New Delhi, Rawat Publications-Institute of Rajasthan Studies : 55-66.

\section{Manuel, Peter}

1993 Cassette Culture: popular music and technology in North India. Chicago, University of Chicago Press.

\section{MARcus, Scott L.}

1993 « Recycling Indian film songs: popular music as a source of melodies for North Indian folk musicians », Asian Music 24(1) : 101-110.

1995 «Parody-Generated texts: the process of composition in biraha, a North Indian folk music genre (India) », Asian Music 26[1] : 95-147.

Neuman, Daniel, Chaudhuri, Shubha et Kothari, Komal

2006 Bards, Ballads and Boundaries: an ethnographic atlas of music traditions in West Rajasthan. Calcutta, Seagull Books

\section{Osmond Smith, David}

1985 Playing on words. A guide to Luciano Berio's Sinfonia. Londres, Ashgate, Royal Musical Association.

\section{Perlman, Marc}

2009 « The limits and dangers of the imagination: rumors of exploitation in the global circuits of traditional music », conférence donnée lors la Journée d'automne de la Société française d'ethnomusicologie, Paris, 12 décembre 2009.
SeEger, Anthony

1996 «Ethnomusicologists, archives, professional organizations, and the shifting ethics of intellectual property $》$, Yearbook for Traditional Music 28 : 87-105.

SeEger, Anthony et ChaUdHuri, Shubha (éd.)

2004 Archives for the future. Global Perspectives on audiovisual archives in the 21st Century. Calcutta, ARCE/AllS, Seagull Books.

\section{Wiliams, Patrick}

2006 « Standards et standardisation. Sur un aspect du répertoire des musiciens de jazz », L'Homme $177-178$ : ?-48.

\section{Discographie}

Rajasthan. A musical Journey. CD, Rupayan Sansthan Archives, Jodhpur, 2003

\section{Ressources électroniques}

\section{BurkHolder, J. Peter (éd.)}

Musical Borrowing. An Annotated Bibliography. Center for the History of Music Theory and Literature, Indiana University; http://www.chmtl.indiana.edu/borrowing.

Critical Public Legal Resources http://www.sarai.net/research/knowledgeculture/critical-public-legal-resources

\section{DOCUMENTATION AND ARCHIVING THE} PERFORMING ARTS

Issues of Intellectual Property Rights and Intangible Cultural Heritage. American Institute of Indian Studies (AISS), Archives and Research Centre for Ethnomusicology (ARCE); http://www.archiving-performance.org

ITwoFS ( Inspirations in Indian Film Songs ")

Music plagiarism. India. oh my god!; http://www.itwofs.com/hindi-others.html.

Liang, Lawrence, Majumder, Atreyee et Suresh, Mayur

2005 «Copyrights/copyleft: myths about copyright »; http://www.sarai.net/research/ knowledge-culture/critical-public-legalresources/sustainingmyths.pdf. 


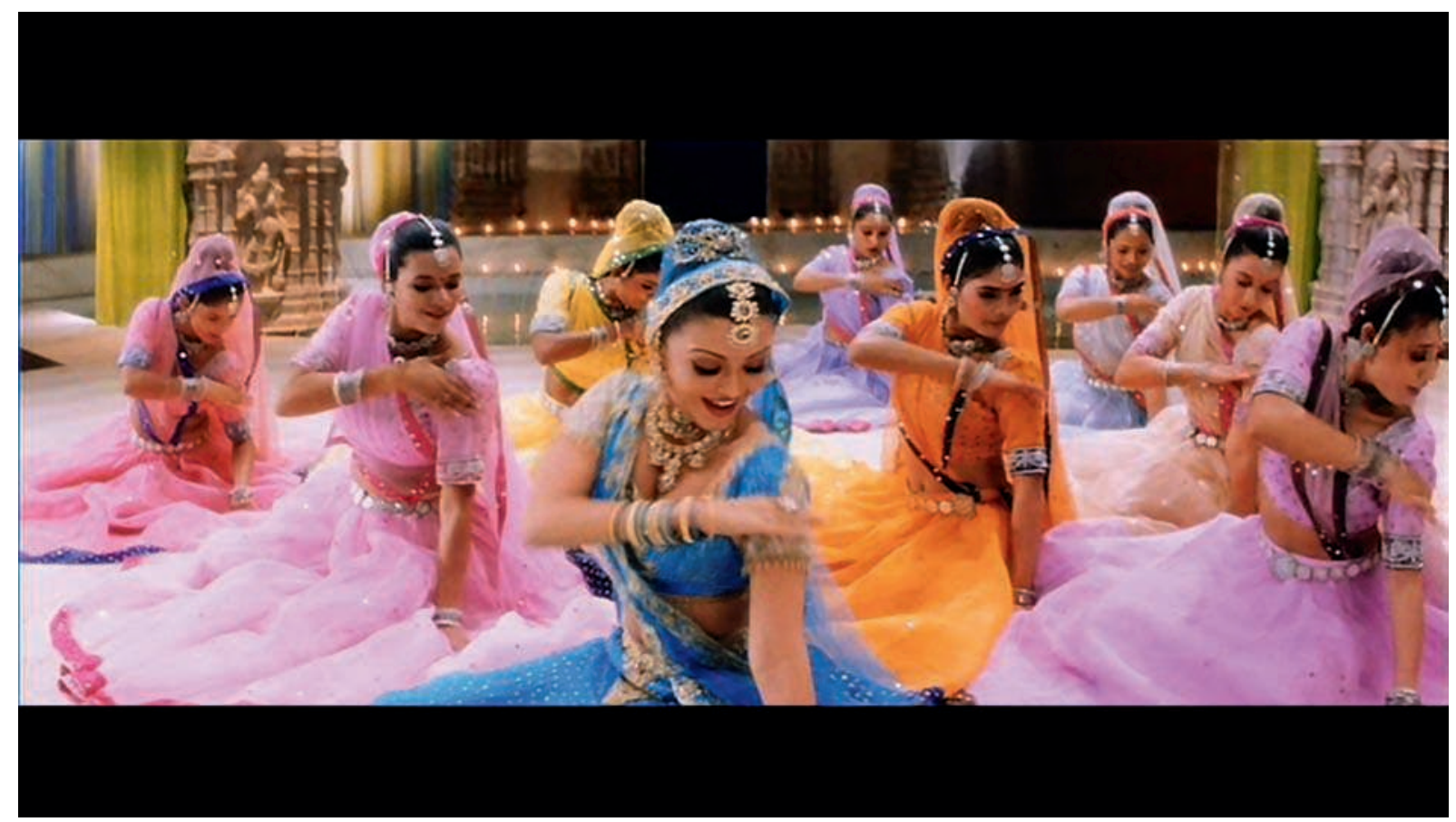

\section{Résumé / Abstract}

Christine Guillebaud, Nimbuda ou la carrière d'un citron amer. Musiques régionales et industrie cinématographique en Inde - II est une procédure de création musicale particulièrement répandue en Inde, l'emprunt de mélodie. II consiste pour le musicien à s'appuyer sur des airs préexistants, et à les investir d'un nouveau contenu, textuel, stylistique, esthétique, etc. C'est dans l'industrie cinématographique qu'il trouve son plus grand aboutissement. La musique filmi s'inspire des genres les plus divers - du rock au hip-hop en passant par la musique symphonique, les traditions classiques indiennes ou encore les nombreux répertoires régionaux. Comment questionner la notion de «propriété intellectuelle » face à ces pratiques cultivant l'emprunt et la multiplicité des sources? Quels sont les ressorts de la copie, de la parodie et du remake ? L'exemple de la chanson Nimbuda, en hindi «Citron amer», fournit un cas exemplaire. Attribuée à la fin des années 1990 à différents «auteurs », tour à tour un chanteur de basse caste manganiyar puis un compositeur de musique de film de Bollywood, la chanson en a par là même hérité une carrière des plus singulières. L'article en retrace la chronologie spécifique et analyse comment les questions de droits d'auteur ont surgi. II montre enfin comment, dans ce marché prolifique où la copie (illégale) des enregistrements est généralisée, les musiciens les plus socialement marginalisés ont bénéficié de nouveaux espaces de diffusion.
Christine Guillebaud, Nimbuda or the Bitter Lemon's Trajectory. Regional Music and the Film Industry in India - The "borrowing" of melodies is a particularly widespread means of musical creation in India. Musicians take pre-existing tunes and give them new lyrics, a new style, a new aesthetic, etc. This technique reaches its acme in the film industry. Filmi music draws on the gamut of styles -from rock to hip-hop, via symphonies, the classical Indian tradition and numerous regional genres. How are we to approach the question of "intellectual property" when faced with these practices that make use of borrowing and draw on multiple sources? And how are we to consider the dynamics of copies, parodies and covers? One clear example of this is the song Nimbuda, Hindi for "Bitter Lemon". Towards the end of the 1990s, the song was attributed to different "authors", first a lower caste Manganiyar bass singer and then a Bollywood film composer. As a result, the song followed a rather singular trajectory, whose precise chronology is mapped out in this article. It also looks at how the issue of copyright has cropped up, and finally shows how within a market where [illegal] copies flourish and recordings are widespread, it is the most socially marginalised musicians who have benefited most from these new forms of distribution. 